latitudes. However, the characteristics cannot be truly determined until tape recordings of them are sent to New Zealand for spectrographic analysis, at the end of the Antarctic winter.

Maximum activity occurred on April 15, 16, 19, 20 and 23 . No consistent diurnal variation in activity has been noted. On all occasions of strong whistler activity the local geomagnetic activity has been moderate or quiet and the ionosphere undisturbed. During an intense auroral display no activity was heard, nor has any correlation been observed with visible meteor showers.

It seems most improbable that the whistlers heard here are propagated along the dipole field passing through Scott Base, as the lines of force in this case would extend to 24 Earth radii above the magnetic equator, and the gyro-frequency would be in the vicinity of $100 \mathrm{c} . / \mathrm{s}$.

Distortion of the dipole field by a strong equatorial ring current could possibly allow whistler propagation along lines of force between conjugate points in high geomagnetic latitudes. This hypothesis, however, does not seem tenable when it is considered that periods of maximum whistler activity at Scott Base have so far occurred during quiet magnetic conditions.

There is no record of lightning discharges being observed in high polar latitudes, and it seems improbable that these could occur as there is insufficient thermal radiation from the Sun to produce the necessary air movements. In these regions there appear to be no natural phenomena capable of initiating whistlers.

During the period that whistlers and 'sferics' have been heard an intense and electrically active lowpressure area has existed some $3,000 \mathrm{~km}$. north of Scott Base. Many of the noises heard could undoubtedly have originated in this area. It is probable that the whistlers heard at Scott Base are propagated from lower latitude regions, first along the appropriate magnetic flux line, and then by reflexion from the lower ionosphere.

Scott Base, Ross Dependency.

L. H. Martin April 30.

\section{Visibility of Noise on Television Pictures}

IN investigations of the visibility of random noise in television pictures ${ }^{1,2}$, it has been found that for narrow-band noise which is just visible the required noise power increases by about $20 \mathrm{db}$. as the centre frequency is raised from 0.15 to $2 \mathrm{Mc} / \mathrm{s}$. For frequencies between 2 and $3 \mathrm{Mc} / \mathrm{s}$., the noise power remains nearly constant ${ }^{1}$, or shows a small fall ${ }^{2}$. It has been suggested ${ }^{1}$ that this may be because of the generation of intermodulation products of lowfrequency noise, due to the non-linearity of the voltage/brightness characteristic of the television tube. These low-frequency products should be much easier to see than the original high-frequency noise. It follows that the non-linearity should also affect visibility of noise if a sinusoidal signal, together with noise, is applied to the tube control-grid. In this case, sum and difference frequencies of the signal and noise should be produced. An experiment which appears to demonstrate the expected intermodulation effect has been carried out.

A normal television raster was displayed on the tube of a television monitor, the monitor being adjusted so that zero-level signal at the video amplifier

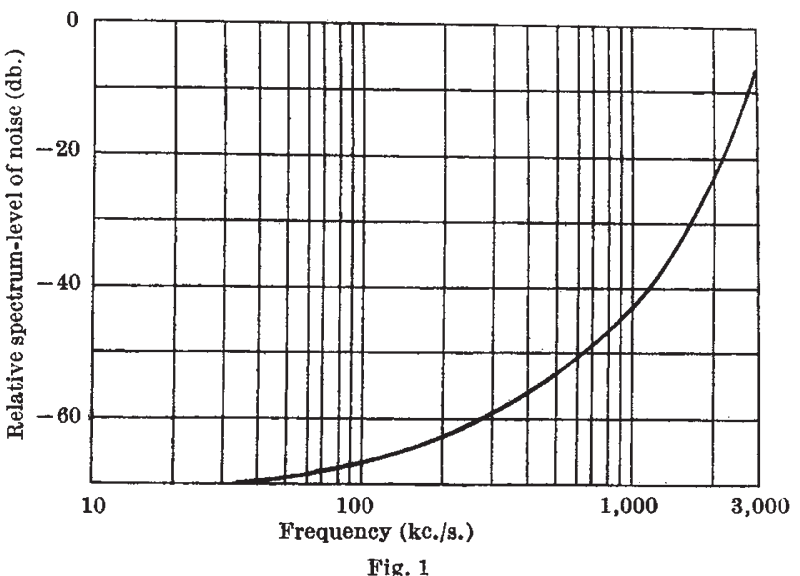

input, that is, 0.7-V. double-amplitude peak, produced a brightness excursion between subjective black and peak white. A sinusoidal signal and random noise were then applied at the video amplifier input. The root-mean-square level of the noise, set at $-40 \mathrm{db}$., was obtained by subtraction of $17 \mathrm{db}$. from oscilloscope measurement of the quasi peak-topeak level ${ }^{1}$ relative to $0 \cdot 7-\mathrm{V}$. double-amplitude peak. This noise-level was quite low, but fairly representative for television. The noise band had a sharp topcut at $3 \mathrm{Mc} . / \mathrm{s}$., and extonded down to about $50 \mathrm{c} . / \mathrm{s}$. The noise was either 'white' (uniform power distribution over the frequency band) or 'quasi-triangular' (power per unit band-width increasing with frequency). The following results were practically independent of tube brightness setting. The visibility of 'white' noise appeared to be unaffected by presence of the sinusoidal signal, although the signal was increased to zero-level, and the frequency varied between 0.1 and $3 \mathrm{Mc} / \mathrm{s}$. However, application of a sinusoidal signal of small amplitude drastically increased the visibility of 'quasi-triangular' noise. Visibility of noise increased with signal-level and, for a fixed level, maximum visibility occurred at a particular signal frequency $f_{8}$. This frequency was between 2.5 and $2.9 \mathrm{Mc}$./s., and increased as the noise spectrum was increasingly peaked at the higher frequencies. As the signal frequency was shifted towards $f_{s}$, from either above or below, the noise became progressively coarser in appearance. This was presumably because the approach of $f_{8}$ to the mean 'carrier' frequency of the noise tended to give the noise envelope as the difference intermodulation product. For the 'quasi-triangular' noise spectrum of Fig. 1, an appreciable increase in visibility of noise was produced by application of $a-40 \mathrm{db}$. signal, $f_{s}$ being $2.9 \mathrm{Mc} / \mathrm{s}$.

Further details of this experiment, and others concerned with the effect of non-linearity on noise in television, will be published elsewhere. I wish to thank the Director of Engineering of the British Broadcasting Corporation for permission to publish this communication, and the head of the Designs Department under whose direction the work was carried out.

\section{R. FatehchaNd}

Department of Numerical Automation, Birkbeck College, University of London, Malet Street, London, W.C.1. April 29.

' Post Offee Radio Report No. 2289 (1955).

2 Maurice R. D. A., Gilbert, M., Newell, G. F., and Spencer, J. G. B.B.C. Engineering Division Monograph No. 3 (1955). 